\title{
Large-area flexible organic solar cells
}

\author{
Fu Yang $\mathbb{D}^{1}$, Yuting Huang ${ }^{1}$, Yaowen $\mathrm{Li} \mathbb{D}^{1,2 \bowtie}$ and Yongfang $\mathrm{Li}^{1,3 凶}$
}

Two major challenges need to be overcome to bridge the efficiency gap between small-area rigid organic solar cells (OSCs) and large-area flexible devices: the first challenge lies in preparing high-quality flexible transparent electrodes with low resistance, high transparency, smooth surface, and superior mechanical properties. Second, the scalable fabrication of thickness-insensitive photoactive layers with low-cost materials is also an essential task. In this review, recent progress and challenges of flexible largearea OSCs are summarized and analyzed. Based on our analysis, strategies and opportunities are proposed to promote the development of stable and efficient flexible large-area OSCs.

npj Flexible Electronics (2021)5:30; https://doi.org/10.1038/s41528-021-00128-6

\section{INTRODUCTION}

Organic solar cells (OSCs) have attracted significant attention for photovoltaic (PV) applications due to their special merits of intrinsic flexibility, light weight, high throughput large-area printing, low cost, and non-toxic raw materials ${ }^{1-5}$. Facing the increasingly mature crystal silicon-based PV market with even higher PCEs and excellent life time (high stability), the OSCs should pave another way to realize their practical application, such as flexible and portable power source, building integrated PV. Toward this goal, two key technologies should be paid enough attentions: (1) the power generation technology of OSCs needs to be cost-competitive with existing technologies, such as fossil fuels and silicon-based solar cells and (2) the OSCs must be manufactured into flexible devices in sustainable techniques. Some cost model calculations of the levelized cost of energy (LCOE) of OSCs with different device structures show that the OSCs can keep up with inorganic PVs, if the requirements of the PV magic triangle (high-efficiency, low-cost, and long-lifetime) could be met ${ }^{6}$.

Since the last several decades, lots of researchers have been working on material development to improve PCE of the OSCS. However, most reported efficient OSCs have been fabricated using the non-scalable spin-coating method ${ }^{7}$. The main merit of the spin-coating technique is that it can easily deposit high-quality organic thin films of well-defined thicknesses and different compositions. However, this method wastes more than $90 \%$ of the solution and generally shows a process limitation on substrate size $^{8}$. In the spin-coating process, the morphology and thickness of organic photoactive layers are determined by the continuous centrifugal force of the spin-coater, which cannot be realized in scalable coating processes and is incompatible with sheet-tosheet (S2S) and roll-to-roll (R2R) high throughput manufacturing. Therefore, the successful commercialization of OSCs requires the development of alternative manufacturing technologies that are scalable and can be easily transferred to S2S or R2R manufacturing, such as blade coating, slot die coating, gravure printing, inkjet printing, and screen printing. However, current performances of the small-area OSCs based on those scalable methods are lower than that based on the spin-coating method, and substantial losses in PCEs occur when transferring the lab cells to large-area
OSCs by scalable fabrication methods. Due to its minimal equipment requirements and little ink consumption, doctor blade coating is often employed as the first step to replace spin-coating method toward a scalable deposition. However, it is hard to adapt to the high throughput printing technology owing to the discontinuous ink supplying. The more complicated coating methods, like slot die coating, gravure printing, inkjet printing, and screen printing, can be used in high throughput printing technology but exhibiting higher equipment requirements. The main shortcoming of those scalable methods is that it is difficult to control uniformity, thickness, crystallization, and morphology. After the scalable coating process, the solvent of ink must evaporate in a controlled manner to leave a stabilized, homogeneous, and desired arranged film. Thus, it is essential to deeply understand the mechanism of organic film formation based on those coating methods to further obtain high-performance largearea OSCs.

Besides, flexible OSCs fabricated on flexible substrates possess the great advantages of the OSCs, which can be applied in portable and wearable power sources. The flexibility is considered to be the most superior feature of the OSCs compared to the silicon-based solar cells. The difference between the flexible OSCs and rigid OSCs is that the substrate of flexible OSCs is polyethylene terephthalate (PET), polyimide, or polyethylene naphthalate (PEN) and the rigid substrate is glass. A typical flexible OSC usually consists of a flexible transparent conductive electrode (FTE), a photoactive layer, two buffer layers, and a top electrode (sliver, aluminum), as shown in Fig. 1a. Figure $1 \mathrm{~b}$ and Table 1 exhibit the efficiency progress of the flexible large-area $\left(\geq 1 \mathrm{~cm}^{2}\right)$ OSCs prepared by main stream coating techniques (spin-coating, slot die coating, doctor blade coating, gravure printing $)^{9-38}$. The highest PCE of large-area flexible OSCs is just $13.61 \%$ with an active area of $1 \mathrm{~cm}^{2}$, while the PCE was decreased rapidly to $2.1 \%$ when the active area was increased to $120 \mathrm{~cm}^{2} 20,21$. However, the certificated PCE of the large-area OSCs fabricated on rigid indium tin oxide (ITO) glass is up to $11.7 \%$ with an active area of $204 \mathrm{~cm}^{2}$ through doctor blade-coating method ${ }^{39}$. The performance of flexible OSCs still lags behind that of rigid devices, which can be due to the higher sheet resistance $\left(R_{\mathrm{sh}}\right)$ of the FTEs comparing to the rigid ITO glass electrode. Therefore, the development of high-

\footnotetext{
${ }^{1}$ Laboratory of Advanced Optoelectronic Materials, Suzhou Key Laboratory of Novel Semiconductor-optoelectronics Materials and Devices, College of Chemistry, Chemical Engineering and Materials Science, Soochow University, Suzhou 215123, China. ${ }^{2}$ State and Local Joint Engineering Laboratory for Novel Functional Polymeric Materials, College of Chemistry, Chemical Engineering and Materials Science, Soochow University, Suzhou 215123, China. ${ }^{3}$ Beijing National Laboratory for Molecular Sciences, Institute of Chemistry, Chinese Academy of Sciences, Beijing 100190, China. ${ }^{凶}$ email: ywli@suda.edu.cn; liyf@iccas.ac.cn
} 
a

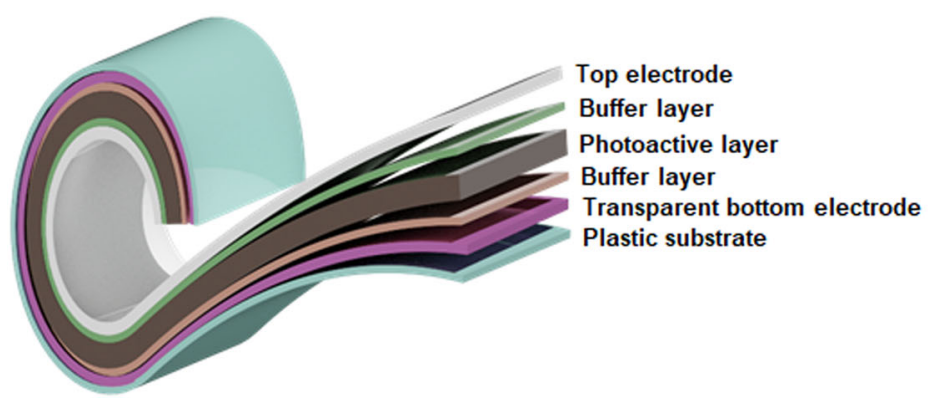

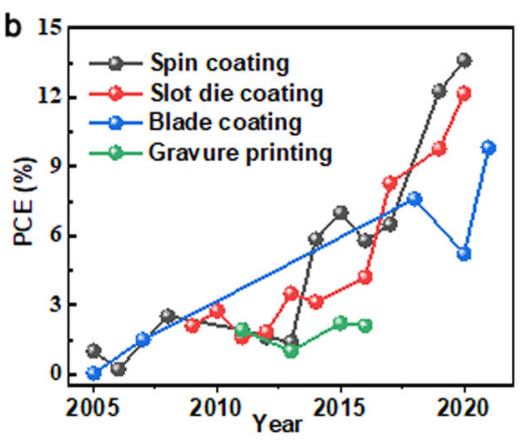

Fig. 1 The development of large-area organic solar cells. a Typical device structure of flexible OSCs. b Efficiency progress of flexible largearea $\left(\geq 1 \mathrm{~cm}^{2}\right)$ OSCs prepared by different coating techniques.

performance large-area FTEs is crucial for accelerating the further application of flexible OSCs.

In this review, the recent progress in materials and scalable technologies for solution-based preparation of large-area flexible OSCs is analyzed. Based on our analysis, the two major challenges in bridging the PCE gap between small-area OSCs and large-area flexible devices are pointed out. The first challenge refers to how to prepare high-quality FTEs on flexible substrates by scalable methods with high reproducibility. The second one consists of selecting and depositing organic photoactive layers by costeffective and eco-friendly scalable methods. Finally, the perspective for upscaling flexible OSCs is discussed.

\section{FLEXIBLE TRANSPARENT ELECTRODES}

An FTE plays a key role in device performance and mechanical flexibility. Low transmittance and low conductivity of the FTEs can lead to optical and electrical losses, thus affecting the performance of the flexible large-area OSCs ${ }^{40,41}$, and the main reasons are as follows: (1) the FTE with low transmittance will reduce the incident light reaching the active layer thus causing optical losses $^{31}$. (2) With increasing the area of OSC, the FTE with high sheet resistance will lead to a higher series resistance $\left(R_{\mathrm{s}}\right)$ of the large-area OSCs, which could dramatically decrease the fill factor (FF) and current density $\left(J_{\mathrm{sc}}\right)^{19,29,42}$ Therefore, the cost-effective FTEs with low sheet resistance and high transmittance are fundamental components and highly needed for large-area flexible OSCs.

ITO is commonly used as the transparent electrode at the rigid glass substrate for efficient lab OSCs because of its low sheet resistance $\left(10 \sim 25 \Omega \mathrm{sq}^{-1}\right)$ and high transmittance (>90\%). However, the performance of OSCs based on ITO FTEs (sheet resistance $10-50 \Omega \mathrm{sq}^{-1}$, transmittance $<85 \%$ ) is much lower than that based on rigid ITO/glass, exhibiting PCEs of only $2.1 \%$ (active area, $120 \mathrm{~cm}^{2}$ ), $4.34 \%$ (active area, $20 \mathrm{~cm}^{2}$ ), 5.25\% (active area, $80 \mathrm{~cm}^{2}$ ), and $8.9 \%$ (active area, $15 \mathrm{~cm}^{2}$ ) for the flexible OSCs based on P3HT-PC ${ }_{61}$ BM, PTB7:PC ${ }_{71}$ BM, SMD2:ITIC-Th, and PBDB-T:ITIC as the active layer respectively ${ }^{30,43-45}$. In addition, owing to the polycrystalline nature of ITO, the mechanical bending and thermal annealing process can shrinkage the flexible substrate, which will cause severe cracking in the ITO film, resulting in a substantial increase in film sheet resistance and diffuse reflection coefficient. Furthermore, the price of ITO is high, and the $R_{\mathrm{s}}$ of flexible OSCs increases as the active area enlarged, limiting its application in large-area flexible $\mathrm{OSCs}^{29}$. Therefore, it is urgently needed to develop alternative flexible transparent electrodes to improve the mechanical stability and efficiency of the large-area flexible OSCs. Until now, many researchers have tried to develop ITO alternatives for realizing the application in large-area flexible OSCs, including ultrathin metal, metal meshes, metal nanowire, carbon materials, conducting polymers, etc.
Ultrathin metal films exhibit a relatively low sheet resistance and excellent flexibility owing to their inherent metal properties. However, increasing the conductivity of ultrathin metal films by increasing the thickness of the metal films would negatively cause their optical loss of transparency. Therefore, the transmittance should be particularly considered when introducing ultrathin metal films into FTEs due to the trade-off between conductivity and transmittance in the large-area flexible OSCs. To solve this issue, Chen et al. used the thickness-gradient ultrathin silver (Ag) film for taking advantage of the high conductivity of the metal film with less light trapping loss, resulting in a PCE of $7.15 \%$ with an active area of $4 \mathrm{~cm}^{2} 17$. They fabricated a thickness stepstructured ultrathin Ag layer (as shown in Fig. 2a) to roughly mimic the efficacy of the thickness-gradient structure, resulting in the optical loss closed to that with $20 \mathrm{~nm}$ homogeneous Ag FTE, but the energy loss is much smaller. As a result, the PCE of large-area flexible OSCs with an active area of $4 \mathrm{~cm}^{2}$ can remain $80 \%$ of that of the rigid OSC with $0.052 \mathrm{~cm}^{2}$ small active area. In addition, thermally evaporated/sputtered metal film suffers from a random nucleation process that metal droplets migrate easily and aggregate randomly on flexible substrate surfaces owing to surface energy mismatch between those two materials ${ }^{46}$. This will lead to the growth of three-dimensional metal islands with a morphology of discrete particles on the substrate, leading to a thicker no-uniform metal electrode with optical loss. Introducing a nucleation-inducing seed layer, such as metal oxide, metal seeds, organic molecules, can provide dense nucleation centers and suppress the growth of large metal islands ${ }^{47-50}$. Lee et al. tried to investigate the role of polymeric nucleation-inducing seed layer in the fabrication of high-quality large-area FTEs through modifying surface energy of plastic substrates with different polymers (polyethyleneimine, poly- $\mathrm{N}$-vinylcarbazole, polyallylamine, polyvinylpyrrolidone, polymethylmethacrylate, and polyethyleneoxide), as shown in Fig. $2 b^{51}$. And they confirmed that the chemical interaction between evaporated metals and functional amines dominates the quality of ultrathin metal films. As a result, largearea FETs $\left(21 \times 29.7 \mathrm{~cm}^{2}\right)$ with high uniformity and superior performance (optical transmittance $>95 \%$ and $R_{\mathrm{sh}}<10 \Omega \mathrm{sq}^{-1}$ ) were obtained through polyethyleneimine modification on the flexible substrate surface.

The low transmittance of the ultrathin metal films is mainly due to their inherent compactness. Metal nanowires and metal grids can provide advantages for balancing the light trapping loss and sheet resistance induced energy loss due to their combined merits of superior optoelectrical properties and good mechanical flexibility. PCEs of $13.61 \%$ for the devices based on PM6:Y6 and $12.16 \%$ for the devices based on PTB7-Th:COi8DFIC:PC ${ }_{71} B M$ were obtained in the large-area flexible OSCs with an active area of $1 \mathrm{~cm}^{2}$ based on $\mathrm{Ag}$ nanowires (AgNWs) and Ag grid as the FTE, respectively ${ }^{20,31}$. Despite their successful applications in large-area flexible OSCs, there are still some critical challenges, such as 
Table 1. The performance progress of flexible large-area $\left(\geq 1 \mathrm{~cm}^{2}\right)$ OSCs prepared by different coating techniques.

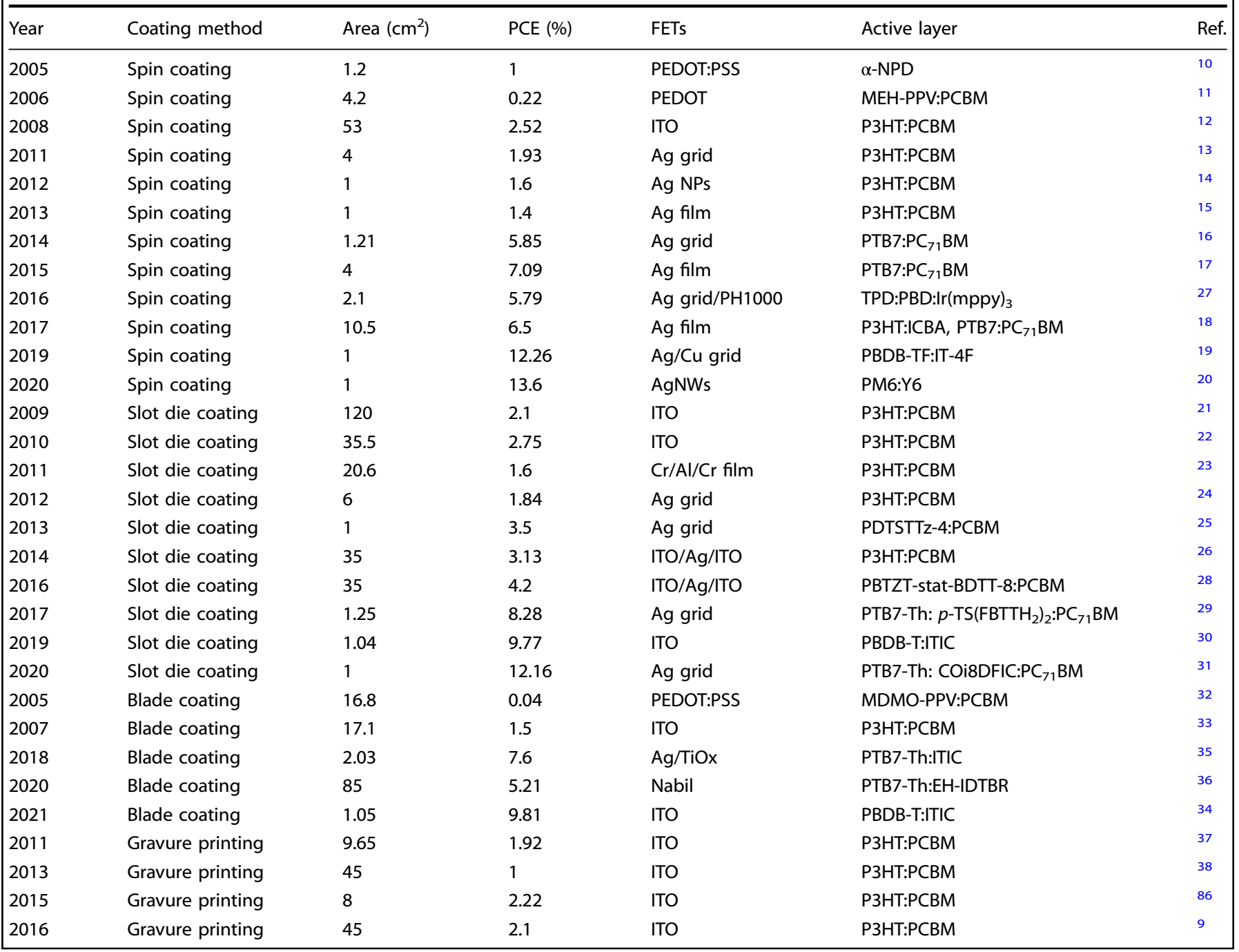

uncontrolled network structure, high contact resistance, and high surface roughness ${ }^{52}$. Researchers have devoted great efforts to solve the above issues, such as doping, adding a protective layer, and post-treatment ${ }^{53,54}$. Chen et al. successfully fabricated gridlike structured AgNWs FTEs with an sheet resistance of $10 \Omega \mathrm{sq}^{-1}$ and a transmittance of around $92 \%$ through using poly(sodium 4styrenesulfonate) (PSSNa) as a polyelectrolyte in the AgNWs disperation, as shown in Fig. $2 \mathrm{c}^{55}$. Li et al. reported $3-\mu \mathrm{m}$-width silver grid embedded in PET substrate, exhibiting very low resistance of $0.5 \Omega \mathrm{sq}^{-1}$ with optical transmittance of $85 \%{ }^{50}$. To make it compatible with large-area flexible OSCs, they have tried to prepare $\mathrm{PET} / \mathrm{Ag}$-grid/PH1000 to improve surface flatness and wettability of the PET/Ag-grid. The PET/Ag-grid/PH1000 FTEs exhibited a resistance of $1.2 \Omega \mathrm{sq}^{-1}$ with an optical transparency of $80 \%$, leading to a PCE of $5.85 \%\left(1.21 \mathrm{~cm}^{2}\right.$ active area) for the device based on PTB7:PC ${ }_{71} B M$ as the photoactive layer, which was the highest performance of the flexible OSCs in that period ${ }^{16}$. However, the parasitic absorption of $\mathrm{PH} 1000$ in the longwavelength region can seriously hinder sunlight utilization. To overcome this issue, they doped 20 wt\% AgNWs into PH1000 for obtaining an optical transparency of $86 \%$ with a sheet resistance of $6 \Omega \mathrm{sq}^{-156}$. In addition, they also tried to remove $\mathrm{PH} 1000$ and prepare a PET/embedded AgNWs substrate (Em-Ag) to address the mismatch problem between the transmittance/absorption spectra of the FTEs as shown in Fig. $2 \mathrm{~d}^{57}$. Owing to the capillary force effect and secondary growth of AZO from solution, the AgNWs parasitic absorption from electrode compositions can be effectively avoided. And the deposited AZO can weld the junction site of the AgNWs. The exposed AgNWs in the underlying Ag-grid further bind with the upper AgNWs layer in the AgNWs:AZO-SG, thus enhancing the mechanical properties of the FTEs. The resultant welding FTEs exhibit sheet resistance of $18 \Omega \mathrm{sq}^{-1}$ with an optical transmittance of $\approx 95 \%$. It needs to mention that the above FETs are also called composite electrodes, which combine metal nanomaterials and other conductive materials, leading to high conductivity and transmittance, smooth surface, high adhesion and coverage, and robust bending durability. This strategy can complement the good properties of each material, which opens more routes for the selection of the conductive material and will be great helpful to promote the development of flexible large-area OSCs.

Carbon nanotubes, graphene, and high conductivity polymers (such as PH1000) have been widely investigated as ITO alternatives on FETs. However, performances of large-area OSCs are still poor due to the low transparency or high sheet resistance of these materials that will not be discussed in this review ${ }^{58}$. 


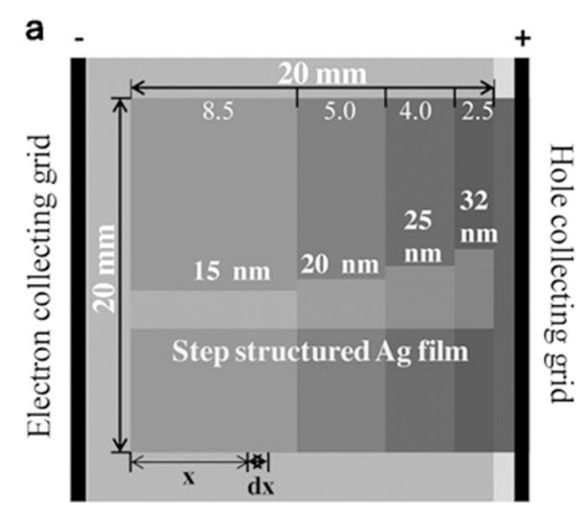

C

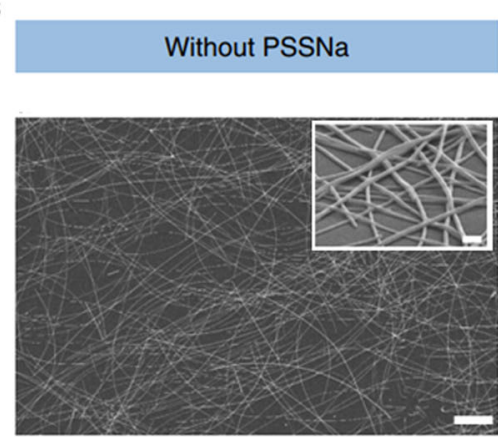

b
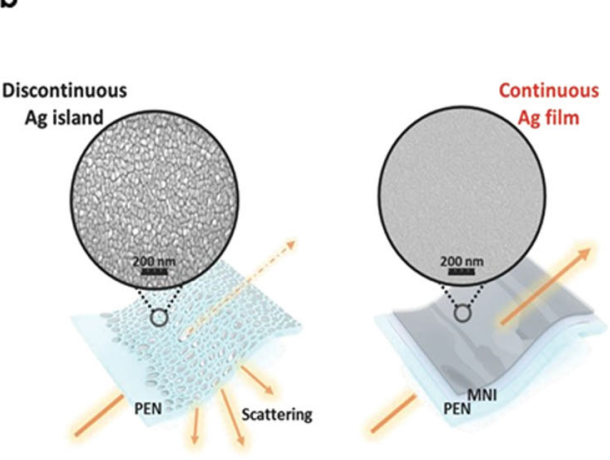

Metal Nucleation Inducers

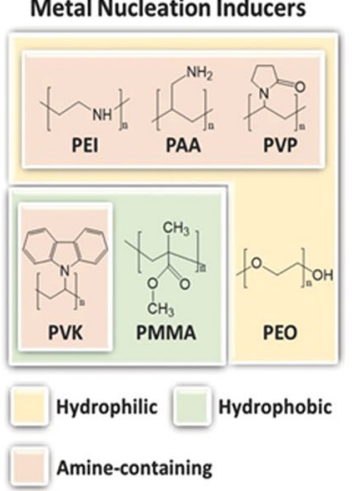

d

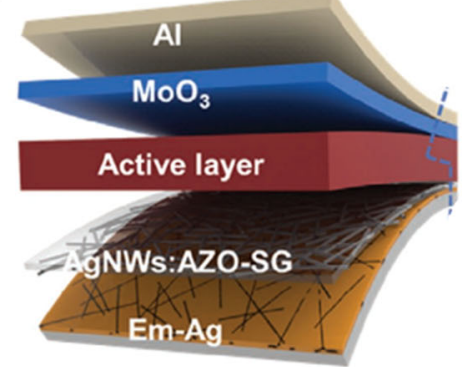

Fig. 2 The development of flexible transparent electrodes for the large-area organic solar cells. a Top view of the structures of the thickness step-structured Ag layer ${ }^{17}$. b Schematic illustration of the ultrathin Ag FTEs and SEM images without and with a polymer nucleationinducing seed layer on PEN substrates. Chemical structures of the polymer nucleation-inducing seed layers ${ }^{51}$. c Surface SEM images of the AgNWs films prepared from the pristine AgNW suspension, AgNW suspension with PSSNa, scale bars, $4 \mu \mathrm{m}$. The inset figures are the crosssection SEM images with scale bars of $200 \mathrm{~nm}^{55,97}$. d Schematic illustration of a flexible OSC based on Em-Ag/AgNWs:AZO-SG FTE ${ }^{57}$.

\section{PHOTOACTIVE LAYER}

The photoactive layer is the most critical component in large-area flexible OSCs. Owing to the intrinsically low charge-carrier mobility and strong exciton binding energy of organic materials, the thickness of the active layer in the most efficient OSCs is $\sim 100 \mathrm{~nm}$. PCEs of large-area flexible OSCs are much lower than that of the small size OSCs, which could be due to the following reasons: (1) the thin active layer is quite sensitive to the point defects on the rough FTEs as lots of point defects exist in the large-area OSCs. (2) The PCE will change significantly if there is a slight thickness variation on the active layer with low thickness insensitivity. (3) It is difficult to prepare a relatively thin organic film by the most scalable coating techniques. The active layer materials, as the most critical functional layer in OSCs, greatly affected these factors and the PCEs of the devices. Herein, we will provide a brief introduction to these state-of-the-art material systems and also discuss the coating techniques for the active layers of the largearea flexible OSCs.

\section{Materials requirement}

Controlling the morphology of the active layers can help to facilitate the charge carriers' generation and transportation in the OSCs. The requirements for the PV materials are as follows: (1) the active layer should be insensitive to the film thickness for the production of efficient large-area flexible OSCs. (2) The organic donors and acceptors need to have regular molecular packing structures with face-on orientation during coating processing to provide efficient charge transfer. (3) Low solubility and strong aggregation tendency for the acceptor materials that can cause an undesirable domain size and low crystallinity need to be avoided during the coating process.
The high mobility of polymer donor can benefit hole transport in the active layer and decreases charge recombination, resulting in high toleration of large thickness variations of the active layer. $\mathrm{P} 3 \mathrm{HT}$ is the main donor material for the large-area OSCs in the early time, due to its high hole mobility $\left(\mu_{\mathrm{h}}, 5 \times 10^{-3} \mathrm{~cm}^{2} \mathrm{~V}^{-1} \mathrm{~s}^{-1}\right)$ and prefer morphology, exhibiting the high insensitivity of film thickness in $\mathrm{OSCs}^{59}$. However, its high-lying highest occupied molecular orbital (HOMO) level results in low $V_{\text {oc }}$ and limited the performance of the OSCs ${ }^{60}$. In addition, the commonly reported conjugated D-A copolymers based on the fluorinated benzothiadiazoles (BT) show limited hole mobility at a level of $10^{-4} \mathrm{~cm}^{2}$ $\mathrm{V}^{-1} \mathrm{~s}^{-1}$. Many researchers have focused their efforts on how to improve the charge mobility and planarity of donors through designing chemical structures ${ }^{61-63}$. Chen et al. reported a D-A copolymer donor $\mathrm{FBT}_{-} \mathrm{Th}_{4}(1,4)$ through using 5,6-difluorobenzothiadiazole as the A-unit and quarterthiophene with solubilizing alkyl chains attached on the two terminal thiophene rings as the D-unit. FBT-Th $4(1,4)$ has a strong interchain aggregation and forms pre-aggregation in a solution at room temperature, leading to hole mobility up to $1.92 \mathrm{~cm}^{2} \mathrm{~V}^{-1} \mathrm{~s}^{-1}$. As a result, FBT-Th $(1,4)$ exhibited high toleration of thickness variations in the OSCs, showing PCEs all over $6.5 \%$ with active layer thickness variations from 100 to $440 \mathrm{~nm}$ and the highest PCE is $7.64 \%$ for the device with $230-n m$ thick active layer ${ }^{64}$. Li et al. developed a low-cost and efficient polymer donor poly[(thiophene)-alt-(6,7-difluoro-2-(2hexyldecyloxy)quinoxaline)] (PTQ10), exhibiting good reproducibility and high tolerance of the film thickness with a PCE over $10 \%$ (PTQ10:IDIC) even at an active layer thickness of $310 \mathrm{~nm}^{65}$. Through combining the post-treatment of thermal annealing and solvent vapor annealing, the blend film exhibited preferred face-on orientation, the closer $\pi-\pi$ stacking, and the higher crystalline characteristics, contributing to balanced charge mobility and less charge-carrier recombination. This could be the 


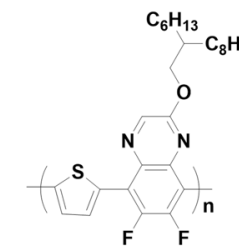

PTQ10

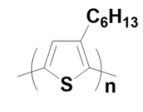

P3HT

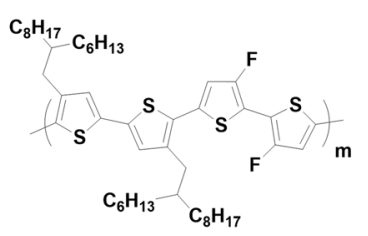

P4T2F-HD

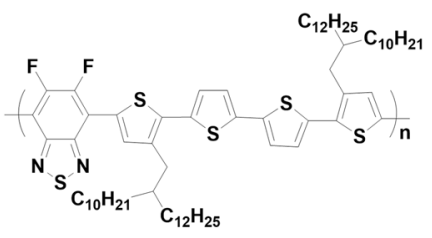

FBT-Th4(1,4)

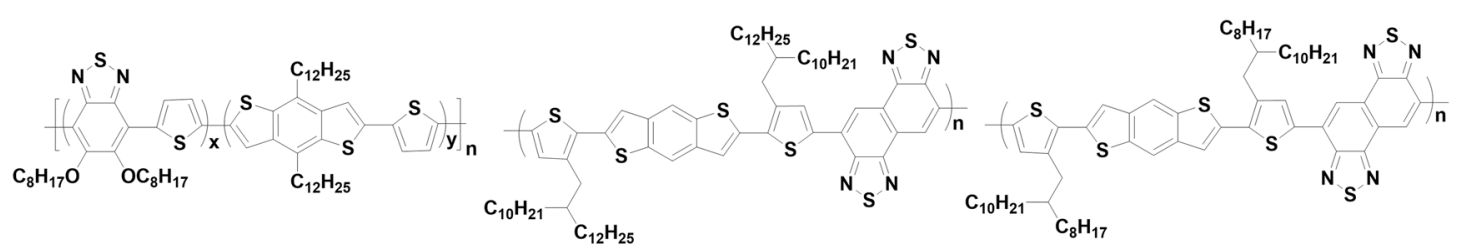

PBTZT-stat-BDTT-8

PNTBDT

PNTT
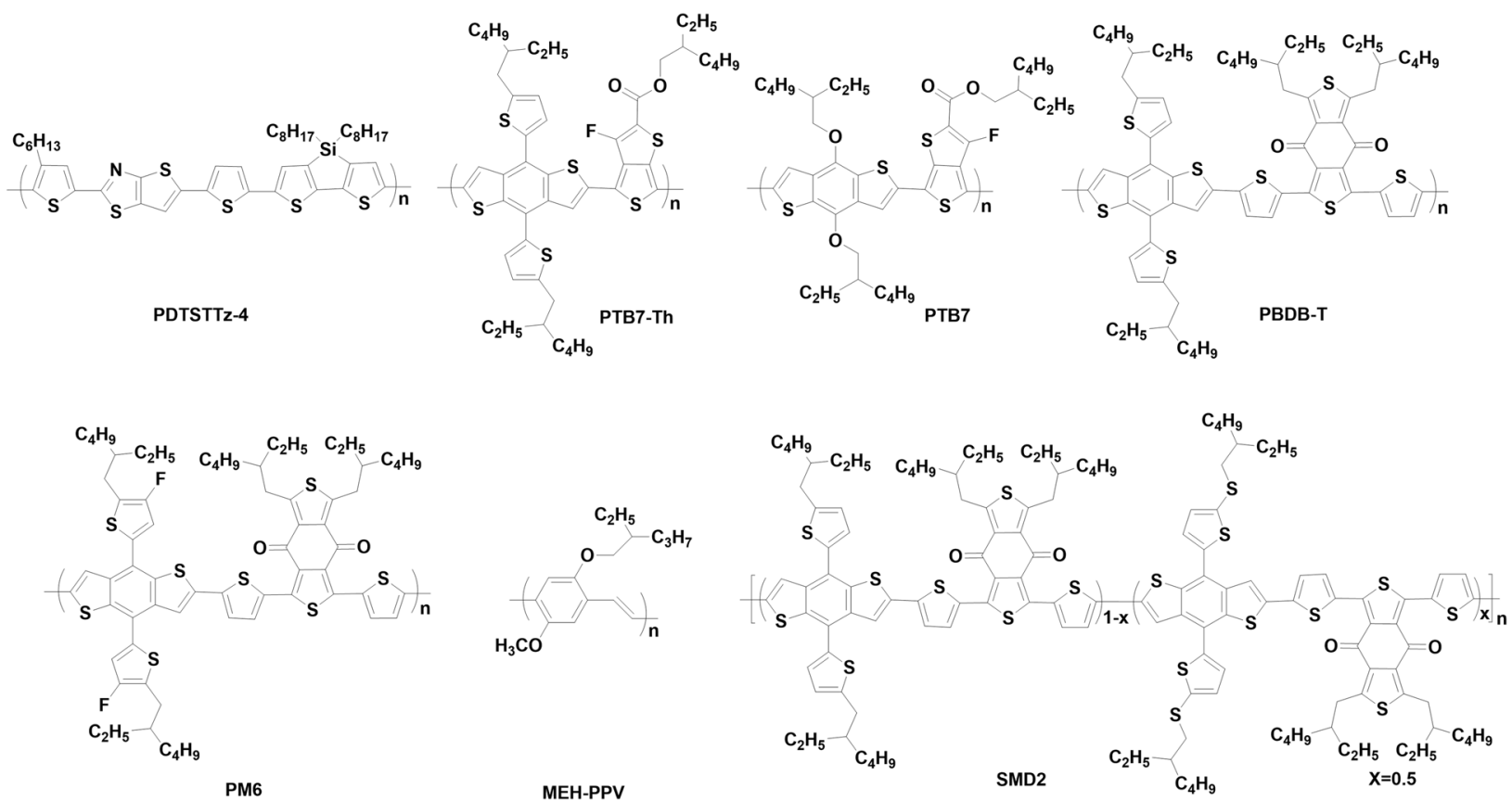

Fig. 3 Donors in the photoactive layer. Chemical structures of polymer donors can be used in large-area flexible OSCs.

reason for the insensitivity of PV performance of the OSCs on their active layer thickness. We also want to emphasize that PTQ10 is one of the few reported active layer materials with low cost and high PCEs.

Compared with BT, naphtho[1,2-c:5,6-c]bis[1,2,5]-thiadiazole (NT)-based polymers, containing two fused BT units, have an enlarged planar aromatic structure, resulting in enhanced interchain packing and thus the improved carrier mobility. Huang et al. developed two $\pi$-conjugated D-A copolymers, PNTT and PNTBDT, containing NT A-units. Both polymers exhibit deep HOMO energy levels and high hole mobilities. OSCs based on PNTT-H (PNTT with high $M_{\mathrm{n}}$ value of $90.1 \mathrm{kDa}$ ) exhibited PCEs greater than $10 \%$ with active layer thicknesses of $150-660 \mathrm{~nm}$ and a PCE of $9 \%$ with an active area thickness of $1050 \mathrm{~nm}$. The PCE insensitive to the active layer thickness is due to the trade-off between light-harvesting capability and charge-carrier transport in the BHJ films ${ }^{66}$. Thieno [3,4-c] pyrrole-4,6-dione (TPD), with strong electron-withdrawing property and coplanar structure, is a very functional A-building block for organic semiconductor materials. The 2,20-bithiophene3,30-dicarboximide unit can be considered as the derivative of the TPD unit, possessing a more expanded conjugated structure, leading to an insensitive active layer thickness for the PV performance of the OSCs ${ }^{67}$.

Even though the non-fullerene acceptors exhibit favorable photoelectric properties and high efficiency in the OSCs, they are quite sensitive in the thick film due to the lower electronic mobility and anisotropic charge transport ${ }^{68,69}$. The emergence of Y6 with high charge-carrier mobilities triggered a breakthrough in the design of non-fullerene receptors, due to their dimer aggregation and three-dimensional charge transport channel ${ }^{70,71}$. Yip et al. used a fluorinated polythiophene derivative (P4T2F-HD) to couple with $\mathrm{Y} 6-\mathrm{BO}$, showing suitable miscibility and can be processed from different nonhalogenated solvents. P4T2F-HD has relatively low miscibility with Y6-BO, which enables both the donor and acceptor materials to form desired packing and nanoscale phase separation morphology for efficient OSCs, leading to small sensitivity to the thickness of the active layer ${ }^{72}$. Through controlling the morphology, Hou et al. successfully achieved highly efficient thickness-insensitive non-fullerene OSCs based on PBDB-T/IT-M as the active layer. Through thermal annealing treatment to control molecular ordering and phase separation, the electron mobility of IT-M becomes higher and the 


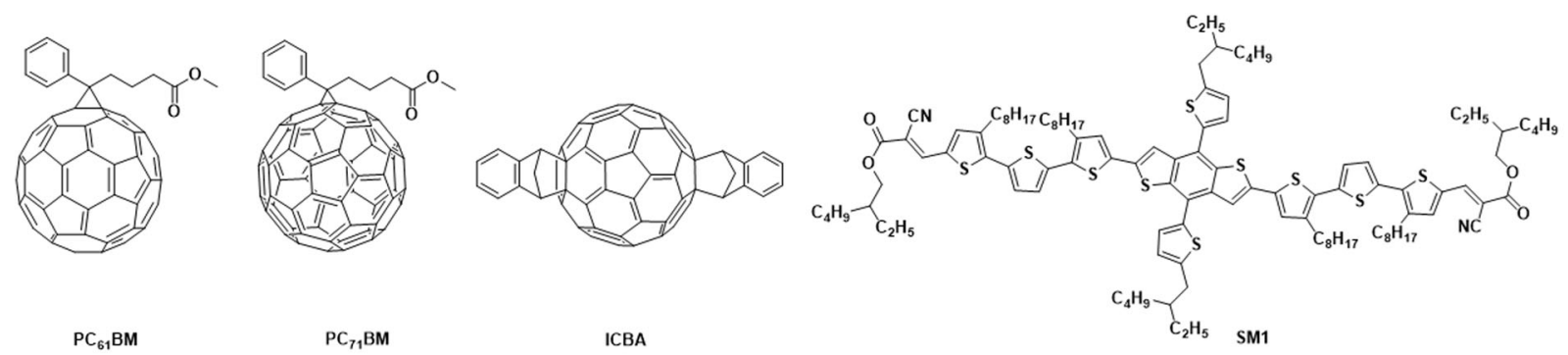

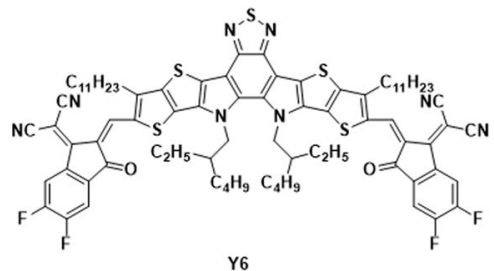

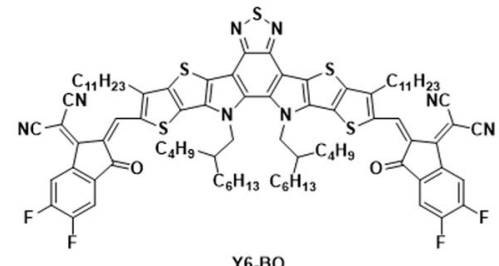

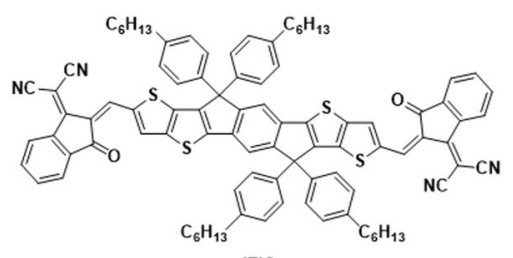

Y6-BO

ITIC
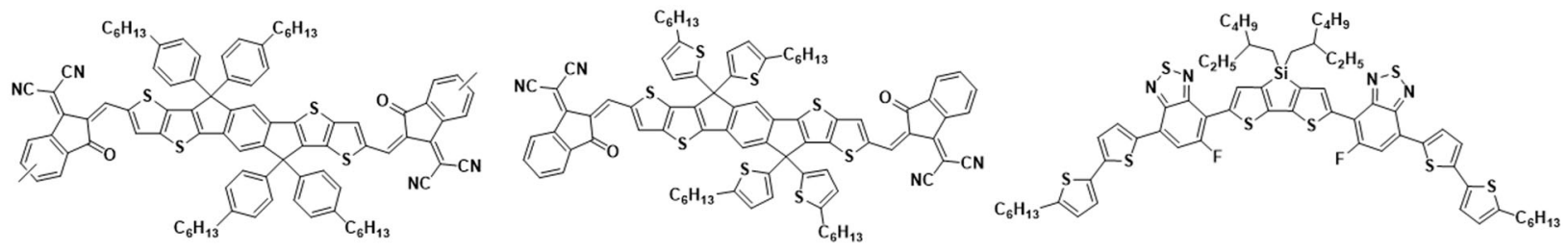

IT-M

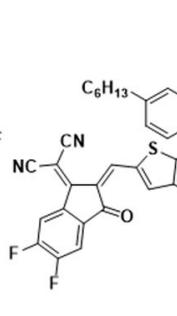

ITIC-Th

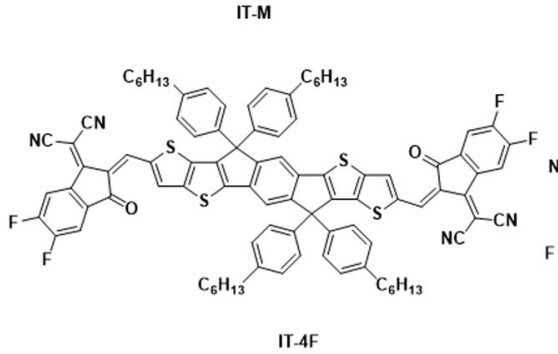

$\mathrm{C}_{6} \mathrm{H}_{13}$

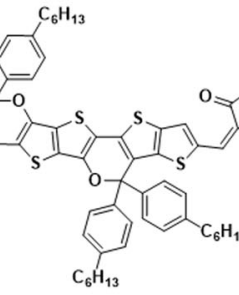

$p$ - DTS $(\text { FBTTH })_{2}$

COIBDFIC

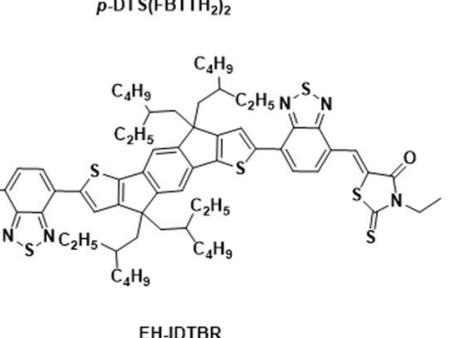

Fig. 4 Acceptors in the photoactive layer. Chemical structures of polymer donors can be used in large-area flexible OSCs.

blend films possess more ordered packing and a higher average domain purity ${ }^{73}$.

Therefore, through tuning the mobility, packing state, the morphology of active layer, high-performance, and thicknessinsensitive PV properties can be successfully realized in large-area OSCs. The chemical structures of the polymer donors and molecule acceptors that can be used in the large-area flexible OSCs are summarized in Figs. 3 and 4.

\section{Coating techniques}

The solution processable OSCs are compatible with R2R production, owing to the chemical nature of organic materials: organic semiconductors are based on abundant and non-toxic raw materials can be tailored for purpose, and the fabrication process is solution processable. Therefore, OSCs have great potential in the high throughput production for enabling competitive values of LCOE. However, most of the state-of-the-art OSCs at laboratory scale are fabricated by the spin-coating method. The superior advantage of the spin-coating method is that it is easy to obtain a high-performed organic active layer with well-defined thicknesses and different compositions. However, the thickness and morphology of the active layer are determined by the continuous centrifugal force, which is unreliable for large-area coating. What is worse, the spin-coating method cannot be applied to the high throughput R2R production. Therefore, researchers need to focus on the alternative methods for scalable coating the OSCs, especially those methods that can be transferred into R2R manufacturing to meet the requirements of commercialization. Blade coating, slot-die coating, gravure printing, and inkjet printing are the most explored methods for preparing the largearea active layers.

The scalable coating process for preparing thin film from solution usually contains three steps: solution wetting, spreading, and film drying. A deep understanding of the three steps will be greatly helpful for getting efficient OSCs. The solution wetting can be divided into static wetting and dynamic wetting ${ }^{74}$. The static wetting describes the injected droplets on the substrate keeping static, which is determined by the thermodynamic equilibrium of gas, liquid, and solid phases ${ }^{75}$. This is related to the interfacial tension of solid-vapor, solid-liquid, and liquid-vapor, which means that the droplet is complete wetting when the horizontal direction and vector of these surface tension components are the same. The static wettability of the solution will directly affect the subsequent film formation ${ }^{76}$. Therefore, the film quality can be regulated by choosing different kinds of solvent and material to tune the wettability of the ink ${ }^{77}$. However, this affection is much smaller than that of dynamic wetting in the upscalable printing methods for the organic active layer due to the continuous liquid flow $^{78}$. Dynamic wetting occurs at the dynamic contact line (the contact line between the meniscus and the substrate), where the air is replaced by the liquid flow ${ }^{74}$. The advancing meniscus is regulated by the surface tension and dynamic pressure. And the receding meniscus directly contacts with the wet film, leading to more influence on the quality of the final film than that of the 
a

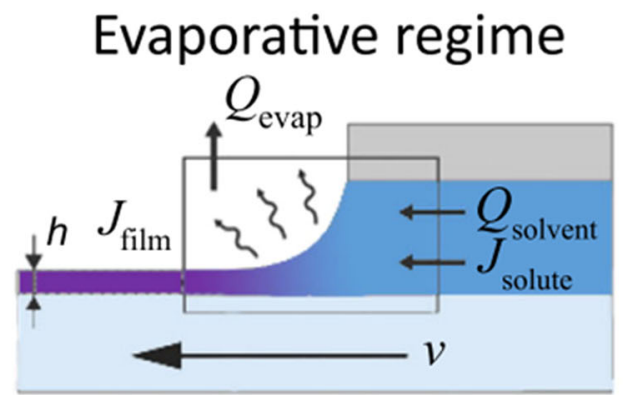

C

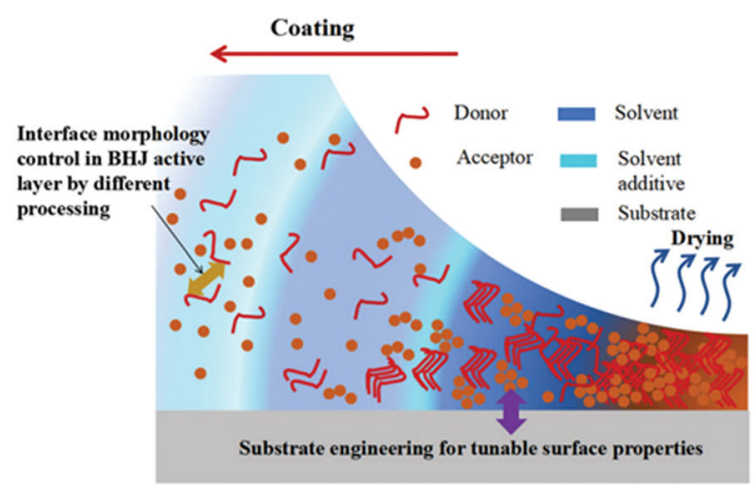

b
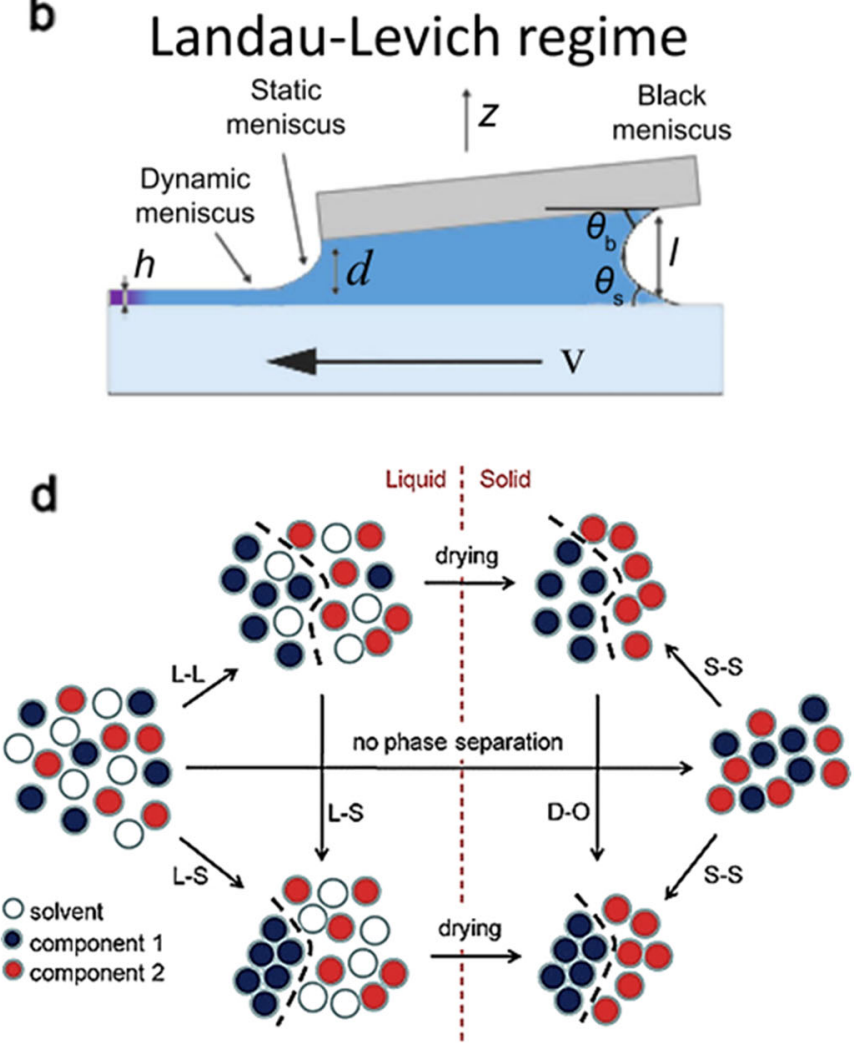

Fig. 5 Mechanism for the scalable coating organic photoactive layers. a, b Schematics of evaporation and Landau-Levich regimes of film deposition ${ }^{79}$. c Schematic depiction of the possible routes to the formation of the BHJ morphology during deposition, which depends on the nature of the substrate and the evolution deposition conditions of the film ${ }^{82}$. $\mathbf{d}$ Schematic exhibition of the phase separation processes that may occur during drying of a solution that contains two components. L-L, L-S, and S-S are liquid-liquid, liquid-solid, and solid-solid-phase separations, respectively, and D-O is disorder-order transition ${ }^{81}$.

advancing meniscus. Following the wetting process, the ink is spread to form a thin film. The thickness of the thin film is mainly determined by two regimes named "evaporation regime" and "Landau-Levich regime" due to the competition of driving forces of solvent evaporation and viscous forces, as shown in Fig. 5a, $b^{79}$. The evaporation regime means that the solvent evaporation speed is faster than the coating speed, showing a thicker film derived from slow coating speed. This is the opposite with Landau-Levich regime region where the solvent evaporation speed is slower than the coating speed, showing thick film derived from the high-speed coating process. The film drying process is usually needed to remove the residual solvent to form the final film. During this process, the post-treatment methods, like antisolvent, annealing, gas quenching, vacuum quenching, can affect the film formation ${ }^{80}$. Different phase separation processes could happen during the drying process that can be schematically exhibited in Fig. $5 \mathrm{c}$, d for the $\mathrm{BHJ}$ film with the donor:accepter system $^{81,82}$. In the following, we will give a detailed discussion of the different scalable coating techniques for preparing the photoactive layers.

Blade-coating method as shown in Fig. $6 \mathrm{a}$ is the most explored method for preparing thin films. During the blade-coating process, the film thickness is determined by the distance between the applicator and substrate, the viscosity of the solvent, the volume of the solvent, blading speed, the concentration of the solution, surface tension, and substrate properties. With low material waste, the efficiency of $16.35 \%$ on the small-area device has been achieved through blade-coating method ${ }^{83}$, which is still lower than that of the spin-coating method. The main reason for this lower performance is due to that there is no continuous centrifugal force to fast evaporate the solvent to well control the film formation of the organic layer. Until now, most of the blade-coated efficient OSCs are based on $\mathrm{BHJ}$ organic active layers and LBL organic active layers. Hou et al. reported a PCE of $7.6 \%$ for the ITO-free flexible larger area OSCs (active area, $2.03 \mathrm{~cm}^{2}$ ) based on PTB7-Th and ITIC as the active layer ${ }^{35}$. Through the incorporation of $0.6 \mathrm{vol} \% \mathrm{DIO}$, the final drying time is prolonged from 72 to $506 \mathrm{~s}$ thus strongly affecting the final morphology of the active layer, resulting in favorable crystallinity, morphology, and internal D/A structure. As a result, the DIO additive helps ITIC molecules migrate from the bottom to the top to form an optimal $\mathrm{D} / \mathrm{A}$ interface distribution in the $\mathrm{BHJ}$ active layer, resulting in the reduced exciton recombination and optimized electrical parameters. A similar result was achieved by $\mathrm{Ma}$ et al. by adding $\mathrm{DIO}$ as the additive into PBDB-T:ITIC BHJ system to adjust film formation, leading to a better performance than that of the spin-coated devices $^{84}$. They claim that the phase separation of the BHJ blend film is more sensitive to the amount of DIO for the blade coating than the spin coating. In addition, the blade-coated OSCs derived from the $0.25 \% \mathrm{DIO}$ additive result in lower diffusion ability of donor and acceptor molecule, demonstrating better stability than the spin-coated device. Recently, Yuan et al. reported regulating crystallization of this PBDB-T:ITIC BHJ film by adding high crystalline FOIC (a fused octacyclic electron acceptor with A-D-A structure) as the additive to form the ternary system ${ }^{34}$. The PBDBT:ITIC:FOIC system cannot only increase the light-harvesting efficiency with complementary absorption, but can also form the acceptor alloy, leading to a highly balanced crystallinity between the donor and acceptor. As a result, a PCE of $9.81 \%$ was obtained on the flexible large-area blade-coated ternary OSCs $\left(1.05 \mathrm{~cm}^{2}\right)$. However, it needs to mention that there are still some limitations for the blade-coating method that are explained as 


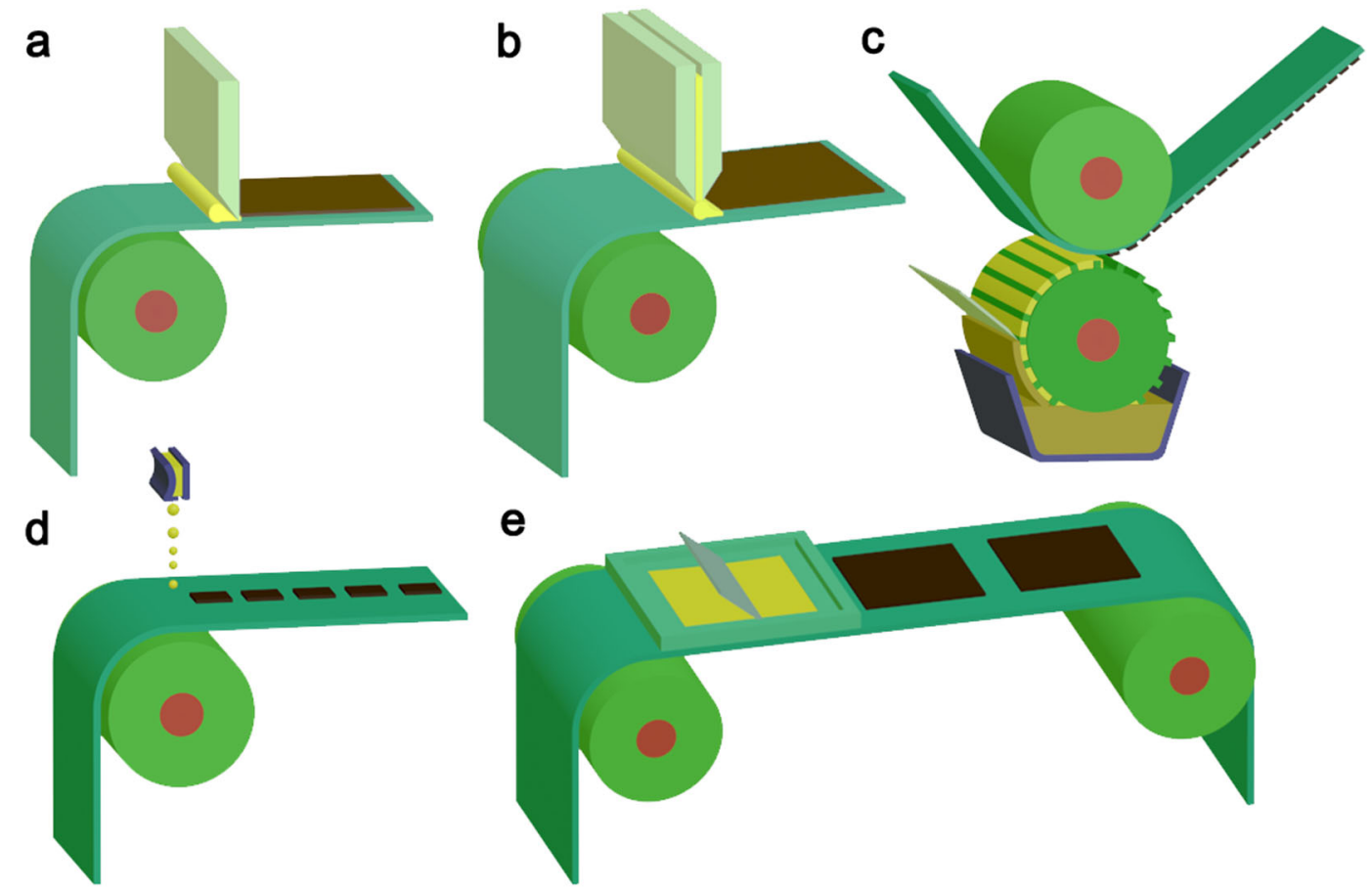

Fig. 6 Common scalable solution deposition methods for large-area flexible OSCs that are compatible with roll-to-roll manufacturing. a Blade coating. b Slot die coating. c Gravure printing. d Inkjet printing. e Screen printing.

follows: (1) there will be a thickness variation for the large-area over $10 \times 10 \mathrm{~cm}^{2}$, as the continuing consumption of solution during the blade-coating progress, leading to the decreased performance. (2) The blade-coating method cannot be used in high throughput continuous production and is difficult to integrate into R2R equipment.

However, the blade-coating method can serve as an analog to the slot die-coating method owing to the similar working mechanism. During the slot die-coating progress as shown in Fig. $6 \mathrm{~b}$, the coating solution is continuously supplied through the slot die head with a pump that rules like the applicator of the doctor blade and capillary force and viscosity of the solution between an applicator and a substrate affect additionally the formation of meniscus. And the thickness of the film can be precisely defined by adjusting the ink-feeding rate and the speed of the slot die head or the substrate. Therefore, the slot diecoating method is considered the most favorable method among researchers for the production of large-area flexible OSCs. However, this method is generally less suitable for applying in the development of high-cost precursors as it usually requires a large amount of the coating solution to fill in the slot to start coating. Many researchers have focused on how to improve film quality to increase the performance of the slot die-coated OSCs, such as high-temperature coating ${ }^{85}$ and gas quenching assistant drying $^{86}$. However, the mechanism of morphological evolution in the photoactive layer is still not deeply understood. Chen et al. demonstrated a general approach to upscale flexible OSCs to the module scale with less performance $\operatorname{loss}^{30}$. They have tried to apply the variable of shear impulse during the film formation process to explain the morphological evolution of the fullerene acceptor-based system (PTB7-Th:PC ${ }_{71} B M$ ) and the non-fullerene acceptor-based system (PBDB-T:ITIC). At last, a transformation factor of shear impulse between spin-coating and the slot-die coating was carried out, which is applicable to various OSCs systems. As a result, for the flexible devices based on PTB7-Th: $\mathrm{PC}_{71} \mathrm{BM}$ and PBDB-T:ITIC, PCEs reached $9.10 \%$ and $9.77 \%$, respectively, for the devices with device area of $1.04 \mathrm{~cm}^{2}$, and $7.58 \%$ and $8.90 \%$, respectively, for the devices with device area of
$15 \mathrm{~cm}^{2}$. Through structuring the devices by ultrashort laser pulses and combining slot die coating into the R2R equipment, Brabec et al. successfully fabricated the large-area flexible module based on the architecture of IMI/ZnO/PBTZT-stat-BDTT-8:PCBM/PEDOT: $\mathrm{PSS} / \mathrm{Ag}$ with a high geometric FF of $98.5 \%$, demonstrating a PCEs of $4.18 \%$ and $3.95 \%$ for the active area of 35 and $98 \mathrm{~cm}^{2}$, respectively ${ }^{28}$.

Gravure printing is a promising method for scaled-up high throughput manufacturing, as shown in Fig. 6c. During gravure printing, the pattern consists of recessed cells below the surface of the roller. These cells are filled with ink, and a doctor blade is used to remove excess ink from the junction between the cells. Gravure printing usually contains three steps: ink filling, wiping, and transfer. The ink, which is applied to the engraved printing cylinder, will be transferred to the substrate. During the printing process, the printing cylinder is partially immersed in the ink container to make the engravings filled with ink. The engraved pattern of the cylinder determines the active area for the ink transfer. A doctor blade is used to remove the excess ink from the inactive area of the printing cylinder. The ink is then transferred from the cylinder to the flexible substrate through applying pressure with an embossing roller. Unlike blade coating or slot-die coating, gravure printing can deposit and pattern a high-precision and high-resolution film in a short time for one time, even for complex patterns. Kopola et al. reported large-area flexible OSCs fabricated with R2R compatible gravure printing method showing PCE of $1.68 \%$ (active area, $15.45 \mathrm{~cm}^{2}$ ) based on P3HT:PCBM as the active layer ${ }^{37}$. Although many research groups have successfully fabricated large-area OSCs based on the gravure printing method, it is not an ideal method for the production of OSCs due to the inherent characteristics of this method: ${ }^{38,87,88}$ (1) The gravure printing method forms dots of the ink on the substrate, whose pattern is the same as the engraved cells. Even the discrete dots can be merged and the variation of wet film thickness can be minimized, the formed thin organic film usually has lots of defects. (2) The coating speed of R2R gravure printing is too high to initially deposit a uniform wet film because the leveling process requires time for the printed solution to spread out ${ }^{9}$. Reverse 
gravure coating is similar to gravure printing but it can produce a uniform film without two-dimensional patterns. Two web guide rolls are introduced along with a coating roll that can touch the web with minimal pressure, leading to the smooth rolling of the web. By applying reverse gravure coating into the R2R manufacturing, Vak et al. fabricated the large-area flexible OSCs showing PCE of $2.1 \%$ (active area, $45 \mathrm{~cm}^{2}$ ) based on P3HT:PCBM as the active layer'.

Inkjet printing is a digitally controlled contact-less patterning technique that can also be equipped into R2R manufacturing as shown in Fig. $6 \mathrm{~d}$. The ink is dispersed by nozzles with fine control of the droplet size and trajectory, leading to a reproducible digital pattern with high resolution and no material loss. The merit of inkjet printing is that it can realize attractive complex or varying patterns of the thin printed film. However, the slow printing speed inhibits the application on printing large-area OSCs. And the viscosity of ink needs to be low enough to form droplets that is also a limitation for its application in $\mathrm{OSCs}^{89}$. The common solvents used for the active layer ink, like chloroform, chlorobenzene, and o-xylene, is typically not applicable for inkjet printing method due to the low viscosities (less than $1 \mathrm{mPas}$ ). Lamont et al. tried to add polystyrene to increase the viscosity ink to enhance the printability with an expense of $20 \%$ of the performance ${ }^{90}$. In addition, high boiling point solvent, such as 1,8 octanedithiol, 1chloronaphthalene, o-dichlorobenzene, can also be used as an additive in the inkjet printing method ${ }^{91}$.

Screen printing can also be induced into R2R manufacturing as shown in Fig. 6e. During the screen printing process, the ink is held and transferred into the substrate by a patterned mesh screen. The thickness of the printed film is defined by the mask thickness, leading to a usual undesired thicker film (1-10 $\mu \mathrm{m})$. And this method has significant limitations regarding the ink properties: (1) to avoid deviation from the pattern, the ink should have high viscosity. (2) To avoid the thickness variation of the thin film raised by the concentration gradients caused by evaporation, the solvent should have low volatility. Until now, the active layer with the composition of P3HT/PCBM has been successfully screen printed for preparing OSCs on the rigid substrates ${ }^{92,93}$.

\section{SUMMARY AND OUTLOOK}

In this review, after a brief discussion of the merits of OSCs, the perspective of challenges to be overcome for upscaling OSCs on a flexible substrate is discussed. Efficiency progress of flexible largearea $\left(\geq 1 \mathrm{~cm}^{2}\right)$ OSCs prepared by different coating techniques is exhibited in Table 1, which indicates the urgency for the development of efficient large-area flexible OSCs. Then the progress and requirement for developing FTEs are discussed to give a clue for preparing the FTEs with low sheet resistance and high transmittance. As the photoactive layer is the most critical component in OSCs, the material requirement for the thickness insensitivity of PV performance is discussed. Then, the upscalable coating methods that can be induced into R2R equipment are discussed, including blade coating, slot die coating, gravure printing, inkjet printing, and screen printing.

Although OSCs have already demonstrated promising potential toward large-area flexible fabrication, many challenges remain.

(1) It still lacks the scalable coating methods for achieving comparable FTEs with low sheet resistance, high optical transmission, smooth surface, and robust bending durability. Recent results indicate that the composite electrodes, which combine metal nanomaterials and other conductive materials, can take advantage of all the merits of those materials to fulfill the requirements for the FTEs, such as ITO/Ag mesh/ ITO, AgNWs:AZO-SG, and Ag-grid/PH1000. In addition, the most efficient results of large-area FTEs are still based on the spin-coating method, which is limited to the substrate size and cannot be directly transferred to the scalable methods. Therefore, it is greatly needed to explore the scalable coating method for the fabrication of the FTEs to deeply understand the coating mechanism of FTEs. In addition, the top electrode is usually deposited by thermal evaporation, which is time consuming and can increase the manufacturing cost of the total OSCs. Development of the techniques for printed top electrodes is a trend for R2R production of large-area flexible OSCs, especially for future commercialization.

(2) Decreasing the thickness sensitivity of the photoactive layer is greatly needed to make the PCEs not change significantly for the slight thickness variation and more compatible with the scalable coating techniques. Tuning the packing state and morphology of the active layer, designing the molecule structure to control the suitable miscibility of the nonfullerene acceptor, and incorporating high hole mobility of donor materials to accelerate hole transportation are effective strategies to achieve the thickness insensitivity of the active layers. In addition, the interlayer materials, especially for the electron transport layer (ETL), are also key factor in the large-area flexible OSCs. The ETL needs to be thin enough $(5-20 \mathrm{~nm})$ to provide good ohmic contact for the charges transferring. And a thick ETL will significantly increase the series resistance of large-area OSCs owing to its low conductivity. Therefore, it is highly necessary to develop materials and related coating techniques for the ETL with thickness-insensitive properties in the large-area flexible OSCs.

(3) Scalable coating techniques are greatly needed for the large-area flexible OSCs. And the thickness precision for blade coating, slot die, inkjet printing, and grave printing is $\mathrm{nm}$, while it is $\mu \mathrm{m}$ for screen printing. The slot die coating method could be the best option for upscaling flexible OSCs as it has low requirements for the materials and solution and can be easily assembled into R2R manufacturing. Inkjet printing is a contact-less method and can print the thin film with fine and accurate patterns, which is a wonderful technique for the scalable fabrication of large-area OSCs regarding future practical applications. But the requirement for the low viscosity of ink will be a limitation of the inkjet printing method. In addition, laminating technology is also one of the promising and effective methods for producing flexible OSCs because it can combine high yield and simultaneous packaging ${ }^{94,95}$. The flexible OSC is made by roll lamination of two parts, referred as the anode side and the cathode side, using the active layer as the adhesive.

(4) The synthetic cost for the donor and acceptor materials should be low enough for the scalable and high throughput fabrication of flexible OSCs due to the huge consumption of those materials. It is essential to address the magic triangle of PV key performance indicators: efficiency, lifetime, and costs (of processing and materials) before further application of the large-area flexible OSCs. Researchers need to spend great effort on exploring low cost, stable, and highperformance donor and acceptor materials. Furthermore, green solvents (nonhalogenated solvent) should be applied in the upscalable coating process, especially the R2R coating process. However, the current performance of OSCs derived from the green solvents is still lower than that derived from the halogenated solvents. Therefore, a deep understanding of the film formation mechanism based on scalable coating methods with green solvents is essential for the instruction for processing optimization and material design. In addition, it is not practical to fabricate a single cell on a large-area substrate because the resistance of the transparent conducting electrode is substantial over a long transport distance. Resistance losses are reduced by dividing a large 
cell into smaller subcells with series interconnections to form a module. And high geometrical fill factor (GFF) is favorable for maximum utilization of the whole device. Currently, the highest GFF of a solar module is up to $99 \%$ by the design of point contact interconnections ${ }^{96}$.

(5) The operation lifetime of OSCs still needs to be improved regarding illumination, air, heating, and bending. Materials design and selection for the layers (active layers and charge transport layers), surface modification, and modification of processing are effective strategies to enhance the stability of OSCs. For example, side-chain engineering is an efficient way to turn the structure of donor materials regarding the stability of OSCs. Besides, encapsulation of OSCs can also effectively prevent the device degradation from oxygen and moisture. The encapsulation materials should be cheap, stable, and easy to deposit, as well as have a high dielectric breakdown, high volume resistivity for oxygen and moisture. In addition, some encapsulation materials can also increase UV illumination and mechanical stability. On the other hand, ITO-free FTEs are also quite important that they should have excellent environmental and mechanical stability and the fabrication process must meet the requirements of largescale preparation. The large-area flexible solar cells also need to show excellent mechanical stability to maintain performance during bending. At last, the module design can also affect the device's stability. Until now, the most efficient reported organic solar modules are based on seriesconnection design. This module design consists of several subcells through P1-P2-P3 etched lines interconnection, resulting in the electrode's direct contact with the organic active layers, which will cause potential concerns for the stability of solar modules due to the generation of shunt paths.

If these challenges can be addressed, the potential application of the flexible OSCs will have a great impact on global energy production and flexible electronic devices.

\section{DATA AVAILABILITY}

All data are available in the main text.

Received: 5 July 2021; Accepted: 19 October 2021; Published online: 04 November 2021

\section{REFERENCES}

1. Liu, F. et al. Organic solar cells with $18 \%$ efficiency enabled by an alloy acceptor: a two-in-one strategy. Adv. Mater. 33, 2100830 (2021).

2. Firdaus, Y. et al. Key parameters requirements for non-fullerene-based organic solar cells with power conversion efficiency $>20 \%$. Adv. Sci. (Weinh.) 6, 1802028 (2019).

3. Li, C. et al. Non-fullerene acceptors with branched side chains and improved molecular packing to exceed $18 \%$ efficiency in organic solar cells. Nat. Energy $\mathbf{6}$, 605-613 (2021).

4. Jin, K., Xiao, Z. \& Ding, L. 18.69\% PCE from organic solar cells. J. Semicond. 42 , 060502 (2021).

5. Riede, M., Spoltore, D. \& Leo, K. Organic solar cells-the path to commercial success. Adv. Energy Mater. 11, 2002653 (2021).

6. Mulligan, C. J., Bilen, C., Zhou, X. J., Belcher, W. J. \& Dastoor, P. C. Levelised cost of electricity for organic photovoltaics. Sol. Energy Mater. Sol. Cells 133, 26-31 (2015).

7. Brabec, C. J. et al. Material strategies to accelerate opv technology toward a gw technology. Adv. Energy Mater. 10, 2001864 (2020).

8. Wang, G., Adil, M. A., Zhang, J. \& Wei, Z. Large-area organic solar cells: material requirements, modular designs, and printing methods. Adv. Mater. 31, 1805089 (2019).

9. Vak, D. et al. Reverse gravure coating for roll-to-roll production of organic photovoltaics. Sol. Energy Mater. Sol. Cells 149, 154-161 (2016).
10. Kushto, G. P., Kim, W. H. \& Kafafi, Z. H. Flexible organic photovoltaics using conducting polymer electrodes. Appl. Phys. Lett. 86, 093502 (2005).

11. Winther-Jensen, B. \& Krebs, F. C. High-conductivity large-area semi-transparent electrodes for polymer photovoltaics by silk screen printing and vapour-phase deposition. Sol. Energy Mater. Sol. Cells 90, 123-132 (2006).

12. Lemaitre, N. et al. High-efficiency large area flexible organic solar cells. Nanoscale Photonic Cell Technol. Photovolt. 7047, 70470 (2008).

13. Galagan, Y. et al. ITO-free flexible organic solar cells with printed current collecting grids. Sol. Energy Mater. Sol. Cells 95, 1339-1343 (2011).

14. Angmo, D., Hosel, M. \& Krebs, F. C. All solution processing of ITO-free organic solar cell modules directly on barrier foil. Sol. Energy Mater. Sol. Cells 107, 329-336 (2012).

15. $\mathrm{Yu}, \mathrm{J}$. S. et al. Transparent conductive film with printable embedded patterns for organic solar cells. Sol. Energy Mater. Sol. Cells 109, 142-147 (2013).

16. Mao, L. et al. Flexible silver grid/PEDOT:PSS hybrid electrodes for large area inverted polymer solar cells. Nano Energy 10, 259-267 (2014).

17. Zuo, L., Zhang, S., Li, H. \& Chen, H. Toward highly efficient large-area ITO-free organic solar cells with a conductance-gradient transparent electrode. Adv. Mater. 27, 6983-6989 (2015).

18. Mao, L. et al. Flexible large-area organic tandem solar cells with high defect tolerance and device yield. J. Mater. Chem. A 5, 3186-3192 (2017).

19. Han, Y. et al. Efficiency above $12 \%$ for $1 \mathrm{~cm}(2)$ flexible organic solar cells with $\mathrm{Ag} /$ Cu grid transparent conducting electrode. Adv. Sci. 6, 1901490 (2019).

20. Wang, Z. et al. High power conversion efficiency of $13.61 \%$ for $1 \mathrm{~cm}^{2}$ flexible polymer solar cells based on patternable and mass-producible gravure-printed silver nanowire electrodes. Adv. Funct. Mater. 31, 2007276 (2020).

21. Krebs, F. C., Gevorgyan, S. A. \& Alstrup, J. A roll-to-roll process to flexible polymer solar cells: model studies, manufacture and operational stability studies. J. Mater. Chem. 19, 5442-5451 (2009).

22. Krebs, F. C., Fyenbo, J. \& Jorgensen, M. Product integration of compact roll-to-roll processed polymer solar cell modules: methods and manufacture using flexographic printing, slot-die coating and rotary screen printing. J. Mater. Chem. 20, 8994-9001 (2010).

23. Zimmermann, B., Schleiermacher, H. F., Niggemann, M. \& Wurfel, U. ITO-free flexible inverted organic solar cell modules with high fill factor prepared by slot die coating. Sol. Energy Mater. Sol. Cells 95, 1587-1589 (2011).

24. $\mathrm{Yu}$, J. S. et al. Silver front electrode grids for ITO-free all printed polymer solar cells with embedded and raised topographies, prepared by thermal imprint, flexographic and inkjet roll-to-roll processes. Nanoscale 4, 6032-6040 (2012).

25. Helgesen, M., Carle, J. E. \& Krebs, F. C. Slot-die coating of a high performance copolymer in a readily scalable roll process for polymer solar cells. Adv. Energy Mater. 3, 1664-1669 (2013).

26. Kubis, P. et al. High precision processing of flexible P3HT/PCBM modules with geometric fill factor over 95\%. Org. Electron. 15, 2256-2263 (2014).

27. Jin, W. Y., Ginting, R. T., Ko, K. J. \& Kang, J. W. Ultra-smooth, fully solutionprocessed large-area transparent conducting electrodes for organic devices. Sci. Rep. 6, 36475 (2016).

28. Lucera, L. et al. Highly efficient, large area, roll coated flexible and rigid OPV modules with geometric fill factors up to $98.5 \%$ processed with commercially available materials. Energy Environ. Sci. 9, 89-94 (2016).

29. Zhang, J. et al. Enhancing performance of large-area organic solar cells with thick film via ternary strategy. Small 13, 1700388 (2017).

30. Meng, X. et al. A general approach for lab-to-manufacturing translation on flexible organic solar cells. Adv. Mater. 31, 1903649 (2019).

31. Wang, G. et al. Synergistic optimization enables large-area flexible organic solar cells to maintain over $98 \%$ PCE of the small-area rigid devices. Adv. Mater. 32, 2005153 (2020).

32. Dennler, G., Lungenschmied, C., Neugebauer, H., Sariciftci, N. S. \& Labouret, A. Flexible, conjugated polymer-fullerene-based bulk-heterojunction solar cells: basics, encapsulation, and integration. J. Mater. Res. 20, 3224-3233 (2005).

33. Tang, $\mathrm{H}$. et al. Highly conducting MXene-silver nanowire transparent electrodes for flexible organic solar cells. ACS Appl Mater. Interfaces 11, 25330-25337 (2019).

34. Zhang, L. et al. Regulating crystallization to maintain balanced carrier mobility via ternary strategy in blade-coated flexible organic solar cells. Org. Electron. 89, 106027 (2021).

35. Lin, Y. B. et al. Printed nonfullerene organic solar cells with the highest efficiency of 9.5\%. Adv. Energy Mater. 8, 1701942 (2018).

36. Jeong, $\mathrm{S}$. et al. Large-area nonfullerene organic solar cell modules fabricated by a temperature-independent printing method. ACS Appl. Mater. Interfaces 12, 41877-41885 (2020).

37. Kopola, P. et al. Gravure printed flexible organic photovoltaic modules. Sol. Energy Mater. Sol. Cells 95, 1344-1347 (2011).

38. Yang, J. L. et al. Organic photovoltaic modules fabricated by an industrial gravure printing proofer. Sol. Energy Mater. Sol. Cells 109, 47-55 (2013). 
39. Distler, A., Brabec, C. J. \& Egelhaaf, H. J. Organic photovoltaic modules with new world record efficiencies. Prog. Photovolt. 29, 24-31 (2020).

40. Xu, G. \& Li, Y. Metal-microstructure based flexible transparent electrodes and their applications in electronic devices. Nano Sel. 1, 169-182 (2020).

41. Cheng, P. et al. Roll-coated fabrication of fullerene-free organic solar cells with improved stability. Adv. Sci. 2, 1500096 (2015).

42. Zhao, Y. et al. A sequential slot-die coated ternary system enables efficient flexible organic solar cells. Sol. RRL 3, 1800333 (2019).

43. Gu, X. D. et al. Roll-to-roll printed large-area all-polymer solar cells with $5 \%$ efficiency based on a low crystallinity conjugated polymer blend. Adv. Energy Mater. 7, 1602742 (2017).

44. Aich, B. R. et al. Ink formulation for organic photovoltaic active layers using nonhalogenated main solvent for blade coating process. Synth. Met. 269, 116513 (2020).

45. Han, Y. W. et al. Evaporation-free nonfullerene flexible organic solar cell modules manufactured by an all-solution process. Adv. Energy Mater. 9, 1902065 (2019).

46. Kang, H., Jung, S., Jeong, S., Kim, G. \& Lee, K. Polymer-metal hybrid transparent electrodes for flexible electronics. Nat. Commun. 6, 6503 (2015).

47. Wang, W. et al. Transparent ultrathin oxygen- doped silver electrodes for flexible organic solar cells. Adv. Funct. Mater. 24, 1551-1561 (2014).

48. Wang, Y. S. et al. Transparent $\mathrm{WO}_{3} / \mathrm{Ag} / \mathrm{WO}_{3}$ electrode for flexible organic solar cells. Mater. Lett. 188, 107-110 (2017)

49. Ghosh, D. S. et al. Highly flexible transparent electrodes containing ultrathin silver for efficient polymer solar cells. Adv. Funct. Mater. 25, 7309-7316 (2015).

50. Li, Y. W. et al. ITO-free photovoltaic cell utilizing a high-resolution silver grid current collecting layer. Sol. Energy Mater. Sol. Cells 113, 85-89 (2013).

51. Jeong, S. et al. Role of polymeric metal nucleation inducers in fabricating largearea, flexible, and transparent electrodes for printable electronics. Adv. Funct. Mater. 27, 1606842 (2017).

52. Sun, Y. et al. Flexible organic solar cells: progress and challenges. Small Sci. 1, 2100001 (2021).

53. Azani, M. R., Hassanpour, A. \& Torres, T. Benefits, problems, and solutions of silver nanowire transparent conductive electrodes in indium tin oxide (ITO)-free flexible solar cells. Adv. Energy Mater. 10, 2002536 (2020).

54. Zhang, Y., Ng, S. W., Lu, X. \& Zheng, Z. Solution-processed transparent electrodes for emerging thin-film solar cells. Chem. Rev. 120, 2049-2122 (2020).

55. Sun, Y. N. et al. Flexible organic photovoltaics based on water-processed silver nanowire electrodes. Nat. Electron. 2, 513-520 (2019).

56. Zeng, G. et al. Breaking $12 \%$ efficiency in flexible organic solar cells by using a composite electrode. Sci. China Chem. 62, 851-858 (2019).

57. Chen, $X$. et al. Realizing ultrahigh mechanical flexibility and $>15 \%$ efficiency of flexible organic solar cells via a "welding" flexible transparent electrode. Adv. Mater. 32, 1908478 (2020).

58. Li, Y. W., Xu, G. Y., Cui, C. H. \& Li, Y. F. Flexible and semitransparent organic solar cells. Adv. Energy Mater. 8, 1701791 (2018).

59. Zeng, L. C., Tang, C. W. \& Chen, S. H. Effects of active layer thickness and thermal annealing on polythiophene: fullerene bulk heterojunction photovoltaic devices. Appl. Phys. Lett. 97, 053305 (2010).

60. Armin, A. et al. Electro-optics of conventional and inverted thick junction organic solar cells. Acs Photonics 2, 1745-1754 (2015).

61. Zhao, J. B. et al. Efficient organic solar cells processed from hydrocarbon solvents. Nat. Energy 1, 15027 (2016).

62. Price, S. C., Stuart, A. C., Yang, L., Zhou, H. \& You, W. Fluorine substituted conjugated polymer of medium band gap yields $7 \%$ efficiency in polymer-fullerene solar cells. J. Am. Chem. Soc. 133, 4625-4631 (2011).

63. Nguyen, T. L. et al. Semi-crystalline photovoltaic polymers with efficiency exceeding $9 \%$ in a $300 \mathrm{~nm}$ thick conventional single-cell device. Energy Environ. Sci. 7, 3040-3051 (2014).

64. Chen, Z. et al. Low band-gap conjugated polymers with strong interchain aggregation and very high hole mobility towards highly efficient thick-film polymer solar cells. Adv. Mater. 26, 2586-2591 (2014).

65. Sun, C. et al. A low cost and high performance polymer donor material for polymer solar cells. Nat. Commun. 9, 743 (2018).

66. Jin, Y. C. et al. Thick film polymer solar cells based on naphtho[1,2-c:5,6-c]bis $[1,2,5]$ thiadiazole conjugated polymers with efficiency over $11 \%$. Adv. Energy Mater. 7, 1700944 (2017).

67. Guo, X. G. et al. Polymer solar cells with enhanced fill factors. Nat. Photonics 7, 825-833 (2013)

68. Chang, Y. L., Zhu, X. W., Lu, K. \& Wei, Z. X. Progress and prospects of thick-film organic solar cells. J. Mater. Chem. A 9, 3125-3150 (2021).

69. Wang, H. L., Chen, L. C. \& Xiao, Y. A simple molecular structure of ortho-derived perylene diimide diploid for non-fullerene organic solar cells with efficiency over 8\%. J. Mater. Chem. A 5, 22288-22296 (2017).

70. Guo, Q. et al. Recent advances in PM6:Y6-based organic solar cells. Mater. Chem. Front. 5, 3257-3280 (2021).
71. Yuan, J. et al. Single-junction organic solar cell with over $15 \%$ efficiency using fused-ring acceptor with electron-deficient core. Joule 3, 1140-1151 (2019).

72. Xiao, J. et al. Surpassing $13 \%$ efficiency for polythiophene organic solar cells processed from nonhalogenated solvent. Adv. Mater. 33, 2008158 (2021).

73. Liu, X. Y. et al. Morphology control enables thickness-insensitive efficient nonfullerene polymer solar cells. Mater. Chem. Front. 1, 2057-2064 (2017).

74. Blake, T. D. The physics of moving wetting lines. J. Colloid Interface Sci. 299, 1-13 (2006).

75. Young, T. III. An essay on the cohesion of fluids. Philos. Trans. R. Soc. London 95, 65-87 (1805).

76. Park, H., Shi, Y. \& Kong, J. Application of solvent modified PEDOT:PSS to graphene electrodes in organic solar cells. Nanoscale 5, 8934-8939 (2013).

77. $\mathrm{Bi}, \mathrm{C}$. et al. Non-wetting surface-driven high-aspect-ratio crystalline grain growth for efficient hybrid perovskite solar cells. Nat. Commun. 6, 7747 (2015).

78. Liu, J., Yu, X., Xue, L. \& Han, Y. Morphology control of polymer thin films. Polym. Morphol. 299-316 (2016).

79. Le Berre, M., Chen, Y. \& Baigl, D. From convective assembly to Landau-Levich deposition of multilayered phospholipid films of controlled thickness. Langmuir 25, 2554-2557 (2009)

80. Zhao, F. W., Wang, C. R. \& Zhan, X. W. Morphology control in organic solar cells. Adv. Energy Mater. 8, 1703147 (2018).

81. Kouijzer, S. et al. Predicting morphologies of solution processed polymer:fullerene blends. J. Am. Chem. Soc. 135, 12057-12067 (2013).

82. Zhang, H., Li, Y. X., Zhang, X. N., Zhang, Y. \& Zhou, H. Q. Role of interface properties in organic solar cells: from substrate engineering to bulkheterojunction interfacial morphology. Mater. Chem. Front. 4, 2863-2880 (2020).

83. Sun, R. et al. A layer-by-layer architecture for printable organic solar cells overcoming the scaling lag of module efficiency. Joule 4, 407-419 (2020).

84. Zhang, L., Lin, B., Hu, B., Xu, X. \& Ma, W. Blade-cast nonfullerene organic solar cells in air with excellent morphology, efficiency, and stability. Adv. Mater. 30, 1800343 (2018).

85. Song, S. et al. Hot slot die coating for additive-free fabrication of high performance roll-to-roll processed polymer solar cells. Energy Environ. Sci. 11, 3248-3255 (2018).

86. Hosel, M., Sondergaard, R. R., Jorgensen, M. \& Krebs, F. C. Fast inline roll-to-roll printing for indium-tin-oxide-free polymer solar cells using automatic registration. Energy Technol. 1, 102-107 (2013).

87. Kapnopoulos, C. et al. Fully gravure printed organic photovoltaic modules: a straightforward process with a high potential for large scale production. Sol. Energy Mater. Sol. Cells 144, 724-731 (2016).

88. Schneider, A., Traut, N. \& Hamburger, M. Analysis and optimization of relevant parameters of blade coating and gravure printing processes for the fabrication of highly efficient organic solar cells. Sol. Energy Mater. Sol. Cells 126, 149-154 (2014).

89. Gertsen, A. S., Castro, M. F., Sondergaard, R. R. \& Andreasen, J. W. Scalable fabrication of organic solar cells based on non-fullerene acceptors. Flex. Print. Electron. 5, 014004 (2020).

90. Lamont, C. A. et al. Tuning the viscosity of halogen free bulk heterojunction inks for inkjet printed organic solar cells. Org. Electron. 17, 107-114 (2015).

91. Eom, S. H. et al. High efficiency polymer solar cells via sequential inkjet-printing of PEDOT:PSS and P3HT:PCBM inks with additives. Org. Electron. 11, 1516-1522 (2010).

92. Jørgensen, M., Hagemann, O., Alstrup, J. \& Krebs, F. C. Thermo-cleavable solvents for printing conjugated polymers: application in polymer solar cells. Sol. Energy Mater. Sol. Cells 93, 413-421 (2009).

93. Zhang, B., Chae, H. \& Cho, S. M. Screen-printed polymer:fullerene bulk heterojunction solar cells. Jpn. J. Appl. Phys. 48, 020208 (2009).

94. Lin, Z. et al. Effect of anode interfacial modification on the performance of laminated flexible ITO-free organic solar cells. Energy Sci. Eng. 9, 502-508 (2021).

95. Bergqvist, J. et al. Asymmetric photocurrent extraction in semitransparent laminated flexible organic solar cells. npj Flex. Electron. 2, 4 (2018).

96. Rakocevic, L. et al. Perovskite modules with $99 \%$ geometrical fill factor using point contact interconnections design. Prog. Photovolt. 28, 1120-1127 (2020).

97. Sun, Y. et al. Flexible high-performance and solution-processed organic photovoltaics with robust mechanical stability. Adv. Funct. Mater. 31, 2010000 (2021).

\section{ACKNOWLEDGEMENTS}

This work was supported by the National Natural Science Foundation of China (grant numbers 51922074, 51820105003, and 22075194), the National Key Research and Development Program of China (grant number 2020YFB1506400), the Natural Science Foundation of the Jiangsu Higher Education Institutions of China (20KJA430010), the Tang Scholar, the Priority Academic Program Development of Jiangsu Higher Education Institutions (PAPD), and Collaborative Innovation Center of Suzhou Nano Science and Technology. 


\section{AUTHOR CONTRIBUTIONS}

F.Y. and Y.T.H. contribute equally to this work. Y.W.L. and Y.F.L. lead the preparation of the manuscript.

\section{COMPETING INTERESTS}

The authors declare no competing interests.

\section{ADDITIONAL INFORMATION}

Correspondence and requests for materials should be addressed to Yaowen Li or Yongfang Li.

Reprints and permission information is available at http://www.nature.com/ reprints
Publisher's note Springer Nature remains neutral with regard to jurisdictional claims in published maps and institutional affiliations. (i) Open Access This article is licensed under a Creative Commons cc) Attribution 4.0 International License, which permits use, sharing, adaptation, distribution and reproduction in any medium or format, as long as you give appropriate credit to the original author(s) and the source, provide a link to the Creative Commons license, and indicate if changes were made. The images or other third party material in this article are included in the article's Creative Commons license, unless indicated otherwise in a credit line to the material. If material is not included in the article's Creative Commons license and your intended use is not permitted by statutory regulation or exceeds the permitted use, you will need to obtain permission directly from the copyright holder. To view a copy of this license, visit http://creativecommons. org/licenses/by/4.0/.

(c) The Author(s) 2021 